\title{
EMA reconsiders 'read-through' drug against Duchenne muscular dystrophy following appeal
}

European drug regulators have recommended a conditional approval for PTC Therapeutics' Translarna (ataluren) to treat Duchenne muscular dystrophy (DMD), just months after rejecting the drug. For the roughly $13 \%$ of boys with DMD whose condition is caused by nonsense mutations, a first disease-modifying treatment is at last around the corner. Clinicians currently prescribe steroids, creatine and angiotensin-converting enzyme inhibitors to ameliorate the symptoms of this muscle-wasting disease caused by a lack of functional dystrophin. PTC's small molecule can restore dystrophin in muscles by making it possible for ribosomes to read through the nonsense codons that stop dystrophin translation. But despite the urgent clinical need, doubts over Translarna's mechanism of action and disappointments in other indications persist as black marks against the drug.

The European regulator's change of heart was unexpected. Stuart Peltz, CEO of South Plainfield, New Jersey-based PTC Therapeutics, says the European Medicines Agency (EMA)'s U-turn was driven by a better understanding of the clinical data. The EMA had rejected Translarna in January in part because the 174-patient phase 2 trial that supported the submission failed to meet its primary endpoint. The trial narrowly missed significance despite a 31.3-meter improvement on the 6-minute walk test (6MWT). PTC Therapeutics appealed the decision, however, and showed that in roughly 40 boys who could walk less than 350 meters at baseline - a recently identified subpopulation of rapidly declining patients-Translarna improved their 6MWT by 68.2 meters compared with placebo. "The scientific advice group said that while the analysis was post hoc, that was a consequence of this being a first test in DMD and that what we did was scientifically and clinically justified," says Peltz. Secondary and tertiary outcomes like muscle function tests, the 10-meter run/walk test, the four-stair climb test, the four-stair decline test and quality-of-life measures also favored Translarna.

The regulators were also swayed by the fact that the biotech's phase 3 confirmatory trial is now nearly fully recruited, Peltz adds, and so is less likely to be derailed (once a drug is commercially available, it can be difficult to recruit and keep patients in a randomized trial in which they might be receiving placebo). The 220-patient confirmatory trial is set to wrap up by the end of 2015. The European Commission must ratify the EMA's decision before the company can start marketing the drug. PTC Therapeutics plans to discuss a US filing with the US Food and Drug Administration (FDA) soon.

Wall Street investors, patient advocates and clinicians are optimistic that the conditional approval is the beginning of a transformation in the DMD space. Two analysts are forecasting average annual Translarna sales of $\$ 1$ billion by 2019 , according to the Thomson Reuters Cortellis database. And shares in the Leiden, Netherlands-based DMD drug developer Prosensa jumped almost $66 \%$, to $\$ 11.03$, in the days after the EMA gave a green light to Translarna. Prosensa's drisapersen is an antisense exon 51-skipping drug for the $13 \%$ of DMD boys whose disease is caused by select deletions in the dystrophin gene. The drug failed to meet its 6MWT efficacy endpoint in a 186-patient phase 3 trial last year, prompting GlaxoSmithKline to bow out of a development partnership with
Prosensa in January. But the EMA's recent opinion on Translarna suggests that a thumbs up could be possible for drisapersen as well. The biotech plans to file for accelerated approval of the drug in the US later this year.

Another competitor in the space, Sarepta, based in Cambridge, Massachusetts, also plans to file for accelerated approval of its exon 51-skipping eteplirsen by the end of the year using data from a 12-patient phase 2 trial. Sarepta took a hit in July, however, when it presented 144-week follow-up data from the tiny trial. Analysts noted a higher rate of decline on the 6MWT than at previous time-points, suggesting that the drug's effect may be starting to wane.

"The approval of ataluren might be a good indication that European regulators now understand DMD and how it progresses," says Marita Pohlschmidt, director of research at the Muscular Dystrophy Campaign, a charity based in London. It may also be a sign that drug developers and clinicians have gotten a grasp on the natural history of the disease, efficacy endpoints and how to perform subgroup and targeted DMD patient analyses, she adds. "It's encouraging. We like what we see," she says.

Parent Project Muscular Dystrophy, a Hackensack, New Jerseybased nonprofit organization, submitted an EMA-inspired DMD drug development draft guidance to the FDA in June so that US regulators could benefit from recent insights (http://www. parentprojectmd.org/ draftguidance). The document, prepared over six months, with input from patients, clinicians, drug developers and regulators, is the first ever patient-initiated draft guidance.

But not everyone is convinced that the Europeans have taken the right approach. "The data showing that dystrophin has been restored is not credible," says Satyakam Bhagavati, a neurologist at SUNY Downstate Medical Center in Brooklyn, New York, who was not involved in the development of the drug. The preclinical data showing that the drug restored dystrophin levels was collected using a PCR-based methodology, he explains, and could therefore be an amplification artifact. "I don't think the clinical trials should have even been done," he says, adding that the same is true of the exonskipping DMD therapies that are also in the clinic. "The functional studies of Translarna have not been good either," he says. "The current approval seems particularly ill advised."

Two groups have also published data suggesting that Translarna's ability to suppress nonsense codons is just an artifact of the firefly luciferase reporter assay that was used to discover the drug (Nat. Biotechnol. 31, 771-773, 2013).

"If you go look at the literature, well over 20 publications demonstrate Translarna's ability to read through nonsense mutations in cell-based and animal models," counters Peltz.

Although a phase 3 trial of the drug in nonsense mutation cystic fibrosis recently failed to hit its primary endpoint, Peltz and his co-investigators speculate that a frequently used inhaled antibiotic might have interfered with Translarna's mechanism of action (Lancet Respir. Med. 2, 539-547, 2014). PTC Therapeutics launched a confirmatory, 208-patient, phase 3, cystic fibrosis trial at the end of June to test this hypothesis and to prepare for a possible future filing.

Asher Mullard Ottawa, Ontario, Canada 